\title{
Analysis of Differentially Expressed Genes in Necrotic Enteritis-infected Fayoumi Chickens using RNA Sequencing
}

\author{
Anh Duc Truong ${ }^{1}$, Yeojin Hong ${ }^{1}$, Jihye Ban ${ }^{1}$, Boyeong Park ${ }^{1}$, \\ Thanh C. Hoang ${ }^{1}$, Yeong H. Hong ${ }^{1}$ and Hyun S. Lillehoj ${ }^{2}$ \\ ${ }^{1}$ Department of Animal Science and Technology, Chung-Ang University, Anseong 17546, Republic of Korea \\ ${ }^{2}$ Animal Biosciences and Biotechnology Laboratory, Agricultural Research Services, \\ United States Department of Agriculture, Beltsville, MD 20705, USA
}

\begin{abstract}
We identified and evaluated differentially expressed genes (DEGs) by RNA-Sequencing (RNA-Seq) in the intestinal mucosa of two Fayoumi chicken lines, M5.1 and M15.2, that are affected by necrotic enteritis (NE); these chicken lines share the same genetic background but have different major histocompatibility complexes. RNA-Seq generated over 49 and 40 million reads for lines M5.1 and M15.2, respectively. The alignment of these sequences with the Gallus gallus genome database revealed the expression of more than 14,500 genes in two lines, among which 581, 1270, and 1140 DEGs were detected when lines M15.2 and M5.1 were compared with the control and compared between each other. The analysis of all DEGs using the gene ontology database revealed annotations for 111 biological processes, 32 cellular components, and 17 molecular functions, and KEEG pathway mapping indicated that the DEGs were primarily involved in immunity, responses to various stimuli, and signal transduction. In addition, we analyzed 183 innate immune genes that were differentially expressed in NE-induced chicken lines, including $46 \mathrm{CD}$ molecular genes, 89 immune-related genes, and $13 \beta$-defensin genes with 3 lineage-specific duplications. Taken together, the transcriptional profiles showed that line M5.1 was more resistant to NE than line M15.2 and that differential gene expression patterns were associated with host genetic differences in resistance to NE. qRT-PCR and RNA-Seq analyses showed that all the genes examined had similar responses to NE (correlation coefficient $\mathrm{R}=0.84$ to $0.88, p<0.01$ ) in both lines. To the best of our knowledge, this is the first study that describes NE-induced DEGs using RNA-seq in two lines with different levels of susceptibility to NE. These results will lead to increased insights on NE disease resistance mechanisms and the role of host genes in the control of the host immune response.
\end{abstract}

Key words: DEGs, Fayoumi chicken, necrotic enteritis, RNA-seq

J. Poult. Sci., 54: 121-133, 2017

\section{Introduction}

Necrotic enteritis (NE), first reported in chickens by Parish (1961), is an enteric disease caused by Clostridium perfringens, a gram-positive, anaerobic, spore-forming, rodshaped bacterium. NE is a global problem and costs the international poultry industry over USD 2 billion per year in production losses and control measures (McReynolds et al., 2009). NE is caused by netB toxin-producing $C$. perfringens type $\mathrm{A}$, and to a lesser extent by type $\mathrm{C}$ strains, and it is a major enteric disease of chickens worldwide (Mot et al., 2013). High-throughput RNA sequencing (RNA-Seq) is a powerful tool used to identify differentially expressed tran-

Received: March 30, 2016, Accepted: October 24, 2016

Released Online Advance Publication: December 25, 2016

Correspondence: Dr. Yeong Ho Hong, Department of Animal Science and Technology, Chung-Ang University, Anseong 17546, Republic of Korea.

(E-mail: yhong@cau.ac.kr) scripts, to classify transcriptomes in different cell types, developmental stages, and conditions, and to detect novel transcripts and genes (Mortazavi et al., 2008; Trapnell et al., 2010, 2012; Wang et al., 2013). Recently, RNA-Seq has been used to analyze genes from different species, including humans (Mortazavi et al., 2008; Wang et al., 2013), mice (Trapnell et al., 2010), Thale Cress (Arabidopsis thaliana) (Lister et al., 2008), cattle (Chitwood et al., 2013), and duck (Huang et al., 2013). In our previous study, transcriptome profiling was used to clarify the molecular mechanisms underlying the pathogenesis of NE and analyze immune gene responses in two inbred chicken lines (6.3 and 7.2) with NE (Truong et al., 2015a, 2015b). However, differentially expressed transcripts in Fayoumi chickens co-infected with Eimeria maxima and C. perfringens have not yet been investigated.

Fayoumi chickens, which originated in Egypt and have been genetically selected into two lines (M5.1 and M15.2), 
have been reported to be resistant to avian leucosis (PinardVan Der Laan et al., 1998). The analysis of genetic diversity in the original M5.1 and M15.2 lines indicated a genetic coefficient of 0.99 with broiler and Leghorn chicken lines (Zhou and Lamont, 1999). These two Fayoumi chicken lines share the same genetic background but have different major histocompatibility complexes (MHC) in the microchromosome (Zhou and Lamont, 1999). Moreover, line M5.1 is more resistant to E. maxima than line M15.2 (Kim et al., 2008, 2009). In particular, the Fayoumi chicken lines show different levels of body weight loss and intestinal lesions upon NE induction, and line M5.1 was more resistant to NE than line M15.2 (Kim et al., 2015). However, the transcriptomes of M5.1 and M15.2 lines with NE have not been evaluated. This study is a RNA-Seq-based genomewide expression analysis of differentially expressed genes (DEGs) in two Fayoumi chicken lines, M5.1 and M15.2, after NE induction to identify host genes associated with resistance to NE. The analysis of the expressed genes in clusters in different functional categories clearly reveals the molecular and cellular events associated with NE.

\section{Materials and Methods}

\section{Experimental Animals and Sample Collection}

The strain of $C$. perfringens (Del-1, $1.0 \times 10^{9} / \mathrm{mL}$ ) and $E$. maxima (41A, $1.0 \times 10^{4} / \mathrm{mL}$ ) was prepared according to protocols of the Animal Biosciences and Biotechnology Laboratory of the Agriculture Research Service, United States Department of Agriculture. Fayoumi chicken lines M5.1 and M15.2 were obtained from Iowa State University. The method used to induce NE was described by Kim et al. (2015). Briefly, 20 chickens were randomly selected and infected with $E$. maxima $\left(1.0 \times 10^{4}\right.$ oocysts/bird $)$ by oral gavage on days 14 and 18 , followed by oral gavage with $C$. perfringens strain Del-1 $\left(1.0 \times 10^{9} \mathrm{CFU} /\right.$ bird $)$. NE was induced according to protocols of the Beltsville Area Institutional Animal Care and Use Committee, United States Department of Agriculture. Intestinal mucosal samples were collected at 20 days post-hatching (Hong et al., 2012).

\section{RNA Extraction and Quality Analysis}

Total RNA was extracted from the intestinal mucosal layer using the TRIzol RNA extraction kit (Invitrogen, Carlsbad, CA, USA), purified using the RNeasy Mini Kit (Qiagen, Germantown, MD, USA), and treated with DNase I (Promega, Madison, WI, USA) according to the manufacturer's instructions. RNA concentration and quality were further determined using the Agilent 2100 bioanalyzer (Agilent Technologies, San Diego CA, USA) and a Tecan F2000 microplate reader (Tecan group Ltd., Männedorf, Switzerland). Samples with RNA integrity $>7$ and high-quality RNA $(28 \mathrm{~S} / 18 \mathrm{~S}>1)$ were used in the experiments.

\section{RNA-Seq, Mapping, and Identification of DEGs}

Total RNA of all samples was pooled from each experimental group before library preparation. The mRNA libraries for Illumina sequencing were constructed using total RNA, as previously described by Trapnell et al. (2010). Sequencing was performed at the Theragen Bio Institute
(Suwon, Korea) using an Illumina HiSeq 2000 highthroughput sequencer, according to the manufacturer's specifications. RNA-Seq data were analyzed according to a method described previously by Trapnell et al. (2012). Briefly, each read was mapped to the Gallus gallus reference genome (v.4.0), which was obtained from the University of California, Santa Cruz (UCSC) database (UCSC: http:// genome.ucsc.edu/), using TopHat v.2.0.3 (http://tophat.cbcb. umd.edu/) and Bowtie v.0.12.8 (http://bowtie-bio.source forge.net/index.shtml), both from Illumina iGenomes (http: //support.illumina.com/). Transcript abundance and differential gene expression were calculated with the program Cufflinks v.2.0.1 (http://cufflinks.cbcb.umd.edu/), as previously described by Trapnell et al. (2012). To identify DEGs, we used the EdgeR package (Robinson et al., 2010). The data were normalized by the fragments per kilobase of exon model per million mapped reads (Mortazavi et al., 2008), and data on reads per kilobase per million reads were used to quantify the relative gene expression. The threshold for considering a gene as a DEG was a false discovery rate $<$ 0.01 . FDR $<0.01$ and $\mid \log _{2}$-ratio $\mid \geq 2$ were used to validate the DEGs.

\section{Expression Pattern, Gene Ontology, and Pathway Enrich- ment Analysis of DEGs}

The genes identified were subjected to hierarchical clustering using Cluster (MeV v4.9: www.tm4.org) and Java Treeview (http://jtreeview.sourceforge.net/). The hierarchical clustering map for the genes in lines M5.1 and M15.2 was prepared using Euclidean distance, with $p$-values $\leq 0.01$. Samples from the intestinal mucosal layer of the two lines were compared as treatment vs. control, respectively. The gene ontology $(\mathrm{GO})$ functional enrichment analysis was performed using Blast2GO (version 2.7.1) (http://www.blast2 go.org/). Kyoto Encyclopedia of Genes and Genomes (KEGG: http://www.kegg.jp/) pathway analysis was performed using DAVID Bioinformatics Resources version 6.7, NIAID/NIH (http://david.abcc.ncifcrf.gov/tools.jsp).

\section{Quantitative Real-Time PCR}

To confirm the differential expression of genes identified by RNA-Seq, quantitative real-time (qRT)-PCR was used for validation. Primer sequences of 19 cytokine genes and an internal control gene (chicken glyceraldehyde-3-phosphate dehydrogenase: GAPDH) were designed using Lasergene software (DNASTAR Inc. Madison, WI, USA) and synthesized by Genotech Co. Ltd. (Daejeon, South Korea; Table S1). Complementary DNA was synthesized using $3 \mu \mathrm{g}$ of total RNA treated with DNase I (Thermo Scientific, Waltham, MA, USA), according to the manufacturer's recommendations. RNA was reverse-transcribed using the Maxima First Strand cDNA Synthesis Kit (Thermo Scientific), according to the manufacturer's recommendations. The gene expression of cytokines was quantitated using standard curves generated using $\log _{10}$-diluted cDNA from individual total RNA, as previously described by Hong et al. (2012). Complementary DNA (100 ng) was added to a reaction mixture containing $10 \mu \mathrm{L}$ of $2 \mathrm{X}$ Power SYBR Green Master Mix (Roche, Indianapolis, IN, USA), $0.5 \mu \mathrm{L}$ of each primer, and 
RNase-free water to a total volume of $20 \mu \mathrm{L}$. qRT-PCR was performed using a LightCycler 96 system (Roche) and a standard cycling program. The relative gene expression was quantitated using the $2^{-\Delta \Delta \mathrm{Ct}}$ method after normalization with GAPDH (Livak and Schmittgen, 2001).

\section{Statistical Analysis}

Statistical analysis was performed using SPSS software version 21.0 for Windows (IBM, Chicago, IL, USA). Data were expressed as mean \pm standard deviation. Comparisons were performed using Student's $t$-test for two-group comparisons, and the level of statistically significant difference was set at $p<0.05$.

\section{Results and Discussion}

\section{Gene Profiling for the NE-afflicted Chicken Lines}

We examined global gene expression profiles using the intestinal mucosa transcriptomes of the two NE-induced Fayoumi chicken lines and controls (Table 1). Approximately 439 million paired-end reads were aligned with the merged reference gene set. After gene mapping to the $G$. gallus reference genome database, 46,030,728, 40,720,918, $49,822,608$, and $52,698,770$ uniquely mapped reads were acquired from the controls and NE-induced lines M15.2 and M5.1, respectively (Table 1). To assess the quality of RNASeq data, several quality-control analyses were performed. The gene coverage method was used to evaluate the RNASeq results. Overall, gene coverage and the distribution of distinct reads in each sample showed similar patterns in four RNA-Seq datasets. The analysis of the overall coverage in line M5.1 indicated that $70 \%$ and $76 \%$ of the genes in the control and NE-induced samples, respectively, covered more than $70 \%$ of the chicken genome (Fig. S1). In line M15.2, more than $73 \%$ of the sequence reads in the control and treated group covered more than $70 \%$ of the chicken genome (Fig. S1). These data indicate the high quality of the RNASeq data and were used for the analysis of gene expression in the chicken lines. In our previous study (Truong et al., 2015a), the transcriptomes of two NE-induced inbred chicken lines (MD-resistant line 6.3 and MD-susceptible line 7.2) provided a total of approximately 38.8 and 40.2 million sequence reads, respectively. Of the total reads, more than
$70 \%$ were successfully mapped on the chicken genome, suggesting that more than 14,500 genes were identified in the intestinal mucosa of lines 6.3 and 7.2. Chitwood et al. (2013) used RNA-Seq to analyze single bovine blastocysts; these authors found that approximately 38 million sequencing reads were generated per embryo and identified the expression of 9,489 known genes. In addition, Lee et al. (2012) reported that, out of approximately 168 million pairs of reads obtained using RNA-Seq from murine hearts, approximately 95 million reads (57\%) were uniquely mapped. In this study, we calculated the genes based on the counted reads mapped to the G. gallus reference genome, and 12,183 and 12,797 genes were identified in the intestinal mucosa of NE-induced lines M5.1 and M15.2, respectively; overall, 14,542 genes were transcribed in four intestinal mucosal layers. In general, the overall gene expression patterns of line M15.2 were similar to those of line M5.1. We identified DEGs in these two chicken lines. The expression of 581 and 1,270 genes was significantly altered in lines M15.2 and M5.1, respectively. In line M15.2, 308 out of 581 DEGs (53\%) were upregulated and 273 out of 581 DEGs (47\%) were downregulated. In line M5.1, 192 out of 1,270 DEGs (15.11 $\%$ ) were significantly downregulated and 1,078 out of 1,270 DEGs $(80.89 \%)$ were significantly upregulated $(p<0.01$, fold-change $\geq 2$; Table 1 and Fig. 1). These findings, together with the hierarchical clustering analysis results, showed the occurrence of more appreciable responses in line M5.1 than in line M15.2 and suggested that 1,140 genes were differentially expressed; in addition, 1065 (93.42\%) DEGs were strongly upregulated in line M5. 1 and $75(6.58 \%)$ DEGs were upregulated in line M15.2 $(p<0.01$, foldchange $\geq 2$; Table 1 and Fig. 1 ). These results clearly indicate that line M5.1 is more resistant than line M15.2.

\section{Innate Immune Responses to NE-induced Chicken Lines}

RNA-Seq analysis provided important information on the differential expression of innate immune genes in the intestinal mucosa of the NE-afflicted chicken lines. In our previous study, we profiled the transcriptome of 149 innate immune-response genes in the NE-afflicted chicken lines 6.3 and 7.2 (Truong et al., 2015a). In this study, we investigated the responses of 183 innate immune genes in the NE-

Table 1. Number of reads and genes detected using RNA sequencing in control and NE induced two chicken lines

\begin{tabular}{|c|c|c|c|c|c|c|}
\hline \multirow{2}{*}{ Group } & \multirow{2}{*}{ Total Reads } & \multirow{2}{*}{$\begin{array}{l}\text { No. of } \\
\text { Expressed } \\
\text { genes }\end{array}$} & \multicolumn{2}{|c|}{$\begin{array}{l}\text { No. of uniquely } \\
\text { mapped reads }\end{array}$} & \multicolumn{2}{|c|}{ No. of DEGs ${ }^{1}$} \\
\hline & & & Genome & Gene & $\begin{array}{l}\text { Versus } \\
\text { Control }\end{array}$ & ${ }^{2}$ DEG set \\
\hline Line M15.2-Control & $103,359,764$ & 12,199 & $46,030,728$ & $37,084,815$ & & \\
\hline Line M15.2-NE & $106,627,968$ & 12,183 & $40,720,918$ & $34,564,640$ & 581 & \\
\hline Line M5.1-Control & $111,112,672$ & 12,330 & $49,822,608$ & $41,633,677$ & & \\
\hline Line M5.1-NE & $119,002,128$ & 12,797 & $52,698,770$ & $44,821,639$ & 1,270 & 1,140 \\
\hline
\end{tabular}




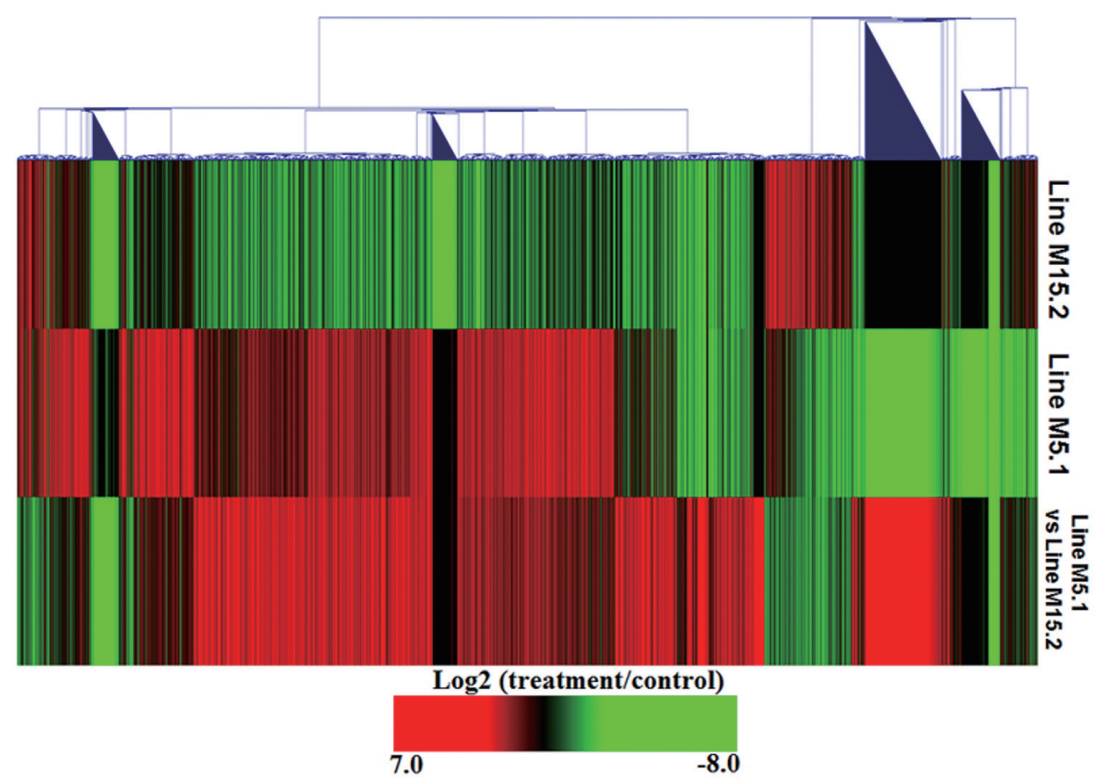

Fig. 1. Heatmap of genes that showed significantly different expression in line M5.1 and M15.2 compared to control and line M5.1 as compared line M15.2-NE infected. This heatmap generated from hierarchical cluster analyses of genes (Euclidean Distance correlation). Genes included in this figure showed significantly different gene expression ( $p<0.01, \log 2$ fold change compared treatment to control). Genes showed in red were up regulated and those showed in green were down regulated in line M5.1 and M15.2 NE induced.

afflicted Fayoumi chicken lines. Herein, the transcriptome analysis of these innate immune genes is presented as fold change in the NE-induced lines compared with the uninfected control (Fig. 2 and Table S2). In line M15.2, the expression of seven genes (IL-22RA2, IL-20RA, IL-5, IL-19, $I L-22, I F I H 1$, and TLR3) significantly decreased by $2.0-$ to 3.6-fold whereas the expression of 11 genes $(I L-20 R B, I L-$ 17REL, TNFRSF13B, TNFAIP2, IL-21, CCLI10, TNFAIP 8L3, TGFBR3, IL-17C, SPP1, and CCR10) significantly increased by 2.3 - to 4.2 -fold compared NE-afflicted chickens with the control ( $p<0.01$, fold-change $\geq 2$; Fig. 2 and Table $\mathrm{S} 2)$. In addition, the expression of five genes $(I L-6, I L-17 F$, CCLI5, IL-19, and CCR10) significantly decreased by 2.02to 6.5 -fold and the expression of 43 genes (IFNG, IL-10, IL17B, IL-17C, IL-3, IL-5, IL-9, TLR15, TLR2-1, TLR7, etc.) significantly increased by 2.0 - to 5.3 -fold in the NE-afflicted line 5.1 ( $p<0.01$, fold-change $\geq 2$; Fig. 2 and Table S2). The comparison of lines M5.1 and M15.2, indicated that the expression of five genes (IL17F, SPP2, CCR10, IL19, and $C C L I 5)$ was downregulated by 2.31 - to 6.34 -fold and the expression of 28 genes (IFNG, IL-22, IL-34,IL-5, TLR3, and $T L R 7$, among others) was significantly upregulated by $2.0-$ to 5.27 -fold ( $p<0.01$, fold-change $\geq 2$; Fig. 2 and Table $\mathrm{S} 2$ ). Therefore, number of innate immune genes expressed in line M5.1 was considerably higher than that in line M15.2.

Th1, Th2, Th17, and pro-inflammatory cytokines have key roles in immune responses to infection (Allam et al., 2011).
Of these cytokines, Th1 cytokine genes $I F N-\gamma, I L-21$, and $I L$ 2 were upregulated in the two chicken lines: $I L-21$ was strongly upregulated by 2.78 - and 2.56-fold in M5.1 and M15.2 lines, respectively ( $p<0.01$, fold-change $\geq 2$; Fig. 2 and Table S2). $I L-3$ and $I L-10$ were significantly upregulated by 2.19 - and 1.54 -fold in line M5.1, respectively, and by 2.62 - and 1.73 -fold in line M15.2, respectively. In contrast, $I L-5$ was downregulated by 3.17 -fold in line M15.2 and upregulated by 5.3-fold in line M5.1 ( $p<0.01$, fold-change $\geq 2$; Fig. 2 and Table S2). These results may indicate that $\mathrm{NE}$ induction results in the expression of Th1 and Th2 cytokines in both lines, and the expression levels of Th1 and Th2 cytokine genes were significantly higher in line M5.1 than in line M15.2. We found that four genes that encode cytokine Th17, including $I L-17 B, I L-17 C, I L-17 R E L$, and $I L-$ $23 R$ in line M5.1, and $I L-17 C$ and $I L-17 R E L$ in line M15.2, were upregulated by 2.3 - to 5.0 -fold ( $p<0.01$, fold-change $\geq 2$; Fig. 2 and Table S2). Therefore, Th17 and pro-inflammatory cytokines were expressed to a greater extent in line M5.1 than in line M15.2. The analysis of the transcription of pro-inflammatory cytokines or receptors in line M5.1 indicated that the expression of five out of six genes in the IL-1 cytokine family $(I L-1 R 2, I L-1 R A P, I L-1 R A P L 1, I L-1 R A P L 2$, and $I L-1 R L 1), I L-18 R 1$, and of 4 out of 29 genes in the TNF family (TNFAIP2, TNFAIPSL3, TNFRSF13B, and TNFRSF $6 B)$ was upregulated by 2.0-to 3.5-fold $(p<0.01$, foldchange $\geq 2$; Fig. 2 and Table S2). In contrast, in line M15.2, 


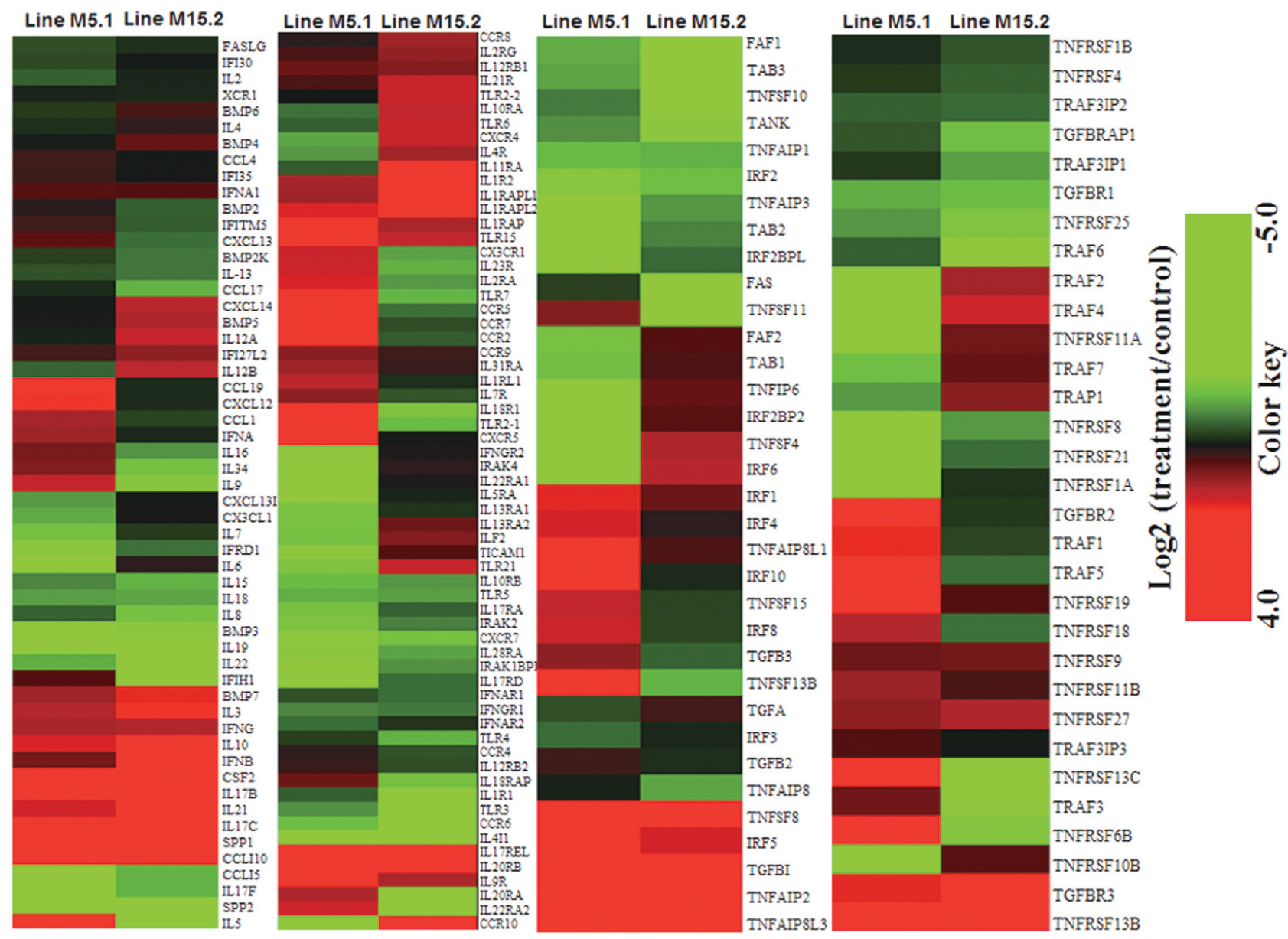

Fig. 2. Hierarchical clusters of $\mathbf{1 8 3}$ innate immune genes responsive to $E$. maxima and $C$. perfringens coinfection in the intestinal mucosa of two chicken lines were based on Euclidean distance correlation analyses. The genes included here showed significant differences in gene expression $(p<0.01, \log 2$ fold change compared treatment to control). The genes shown in red were upregulated and those in green were downregulated.

the expression of pro-inflammatory cytokines changed by -1.7- to 1.6 -fold in the IL-1 cytokine family and 1.3 -fold in IL-18R1; in addition, 3 out of 29 genes of the TNF family (TNFAIP2, TNFAIP8L3, and TNFRSF13B) were upregulated 2.7 to 3.2 -fold (Fig. 2 and Table S2). In our previous study, the expression of Th1, Th2, Th17, and pro-inflammatory cytokines was higher in resistant line 6.3 compared with susceptible line 7.2 in response to NE (Truong et al., 2015a). These results suggest that these cytokines may play an important role in different immune responses to NE in lines M5.1 and M15.2.

Similarly, the transcription analysis of genes of the TLR family indicated that the expression of TLR3 was significantly downregulated by 2.0 -fold $(p<0.01)$ in line M15.2 whereas the expression of TLR7, TLR15, and TLR2-1 significantly increased by 2.67- to 3.50-fold in line M5.1 $(p<0.01$; Fig. 2 and Table S2). Chemokines also play an essential role in the immune response to pathogen infections (Kaiser et al., 2005; Khawli et al., 2008). The transcriptome analysis of 23 chemokines, including C-C motif, C-X3-C motif, and C-X-C motif, indicated that the expression of nine chemokines (six genes encoding $\mathrm{C}-\mathrm{C}$ motif, one gene encoding $\mathrm{C}-\mathrm{X} 3-\mathrm{C}$ motif, and two genes encoding $\mathrm{C}-\mathrm{X}-\mathrm{C}$ motif) was markedly upregulated by 2.0 - to 5.0 -fold and the expression of two chemokines (CCLI5 and CCR10) was significantly downregulated by 2.4 - to 3.6 -fold in line M5.1 $(p<0.01$, foldchange $\geq 2$; Fig. 2 and Table S2). Unexpectedly, the expression of only two chemokine genes (CCLI10 and CCR10) significantly increased by 3.06- and 4.15-fold in line M15.2, respectively $(p<0.01$, fold-change $\geq 2$; Fig. 2 and Table $\mathrm{S} 2$ ).

\section{Avian $\beta$-defensin Genes Are Differentially Expressed to NE-induced Chicken Lines}

Defensins are small cysteine-rich cationic peptides with a 


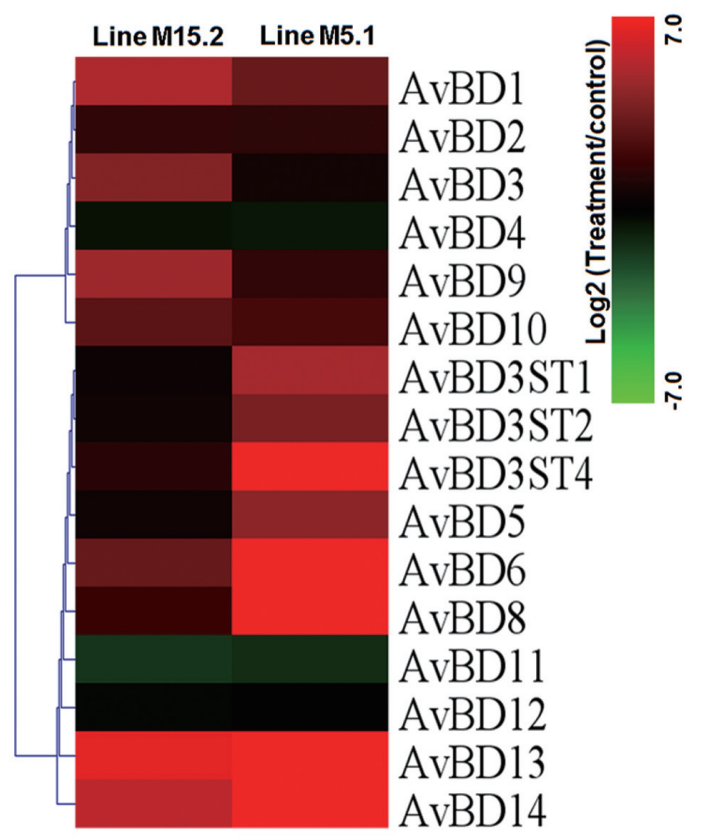

Fig. 3. Identification of $\beta$-defensins family genes responsive of two chicken lines to $\mathrm{NE}$ induced in the intestinal mucosa. The genes included here showed significant differences in gene expression $(p<0.01, \log 2$ fold change compared treatment to control). Genes shown in red was up regulated, and those shown in green was down regulated in chicken lines relative to control.

strong antimicrobial activity against bacteria, fungi, and viruses (Lehrer and Ganz, 2002). Avian $\beta$-defensins play a significant role in the avian innate defense system (Sugiarto and $\mathrm{Yu}, 2004)$. To date, $14 \beta$-defensin genes have been described in chickens: gallinacin (GAL) 1, 1A, and 2 to 13 (Lynn et al., 2007; van Dijk et al., 2008; Hong et al., 2012), and $14 \beta$-defensin genes with six lineage-specific duplications (LSDs) of $A v B D 3$ ( $A v D B 3 A-F)$ were identified in duck (Huang et al., 2013). In this study, transcriptome analysis indicated that 13 avian defensin genes $(A v D B 1$ to $A v D B 14$, except $A v B D 7)$ and three LSDs of these genes ( $A v D B 3 S T 1$, $A v D B 3 S T 2$, and $A v D B S T 4$ ) were expressed in the two chicken lines. Of these, $12 \beta$-defensin genes were markedly upregulated in at least one chicken line compared with the control ( $p$ $<0.01$; Fig. 3). Similar to a previous study that used commercial chickens, $A v D B 2, A v D B 4, A v D B 11$, and $A v D B 12$ were significantly downregulated $(p<0.01)$ in the two chicken lines compared with the controls (Fig. 3). The comparison of the two lines indicates that the expression of $6 \beta$-defensin genes ( $A v B D 3 S T 1, A v B D 3 S T 4, A v B D 6, A v B D 8, A v B D 13$, and $A v B D 14$ ) significantly increased by $2.1-$ to 5.58 -fold in M5.1 line $(p<0.01$, fold-change $\geq 2)$ whereas the expression of $4 \beta$-defensin genes $(A v B D 1, A v B D 3, A v B D 9$, and $A v B D 10)$ significantly increased by 2.0 - to 2.2 -fold in line M15.2 ( $p<0.01$, fold-change $\geq 2$; Fig. 3). In our previous study, we measured the $\beta$-defensin expression in the jejunum and found that $A v B D 8, A v B D 10$, and $A v B D 13$ were highly expressed whereas $A v B D 1, A v B D 6, A v B D 9, A v B D 11$, and $A v B D 12$ were moderately expressed in chickens with NE. In addition, $\beta$-defensin genes were differentially expressed in commercial chickens in response to NE, which indicates that $\beta$-defensins play an important role against NE (Hong et al., 2012). Our results suggest that more $\beta$-defensin genes are expressed in line M5.1 than in line M15.2 and that line M5.1 is more resistant to NE than line M15.2.

\section{Expression of CD molecules in the NE-induced Chicken Lines}

CD molecules are antigens present in the cell membrane during cell development (Stefanova and Horejsi, 1991; Zola et al., 2007). Most CD antigens are involved in immune functions. We investigated the gene expression patterns of $46 \mathrm{CD}$ molecules in the intestinal mucosa of the two lines using RNA-Seq data. In line M5.1, $12 \mathrm{CD}$ genes (CD4, $C D 1 D, C D 226, C D 2, C D 8 B, C D 72, C D 200, C D 28, C D 274$, $C D 1 B, C D 79 B$, and $C D 180$ ) were upregulated by $2.1-$ to $3.8-$ fold whereas $C D 36$ was downregulated by 2.3 -fold $(p<0.01$, fold-change $\geq 2$; Fig. 4 and Table S3). By contrast, in line M.15.2, the expression of 5 genes (CD180, CD86, CD14, CD $40 L G$, and $C D 9$ ) was strongly downregulated by 2.1 - to 5.0 fold whereas the expression of 2 genes (CD34 and CD72) significantly increased by 2.5 - and 2.8 -fold ( $p<0.01$, foldchange $\geq 2$; Fig. 4 and Table S3). The comparison between these chicken lines indicated that $7 \mathrm{CD}$ genes $(C D 72, C D$ 109, CD4, CD300LD, CD180, CD79B, and CD9) were upregulated by 2.4 - to 3.4 -fold ( $p<0.01$, fold-change $\geq 2$; Fig. 4 and Table S3).

Interestingly, $C D 4, C D 8 B, C D 28$, and $C D 72$, which were significantly upregulated in line M5.1, form a co-receptor that assists the $\mathrm{T}$ cell receptor in communicating with an antigen-presenting cell and activating T-cells (Ansari-Lari et al., 1996; Suzawa et al., 2007). The expression of CD72 increased in both chicken lines; CD72 protein is associated with the cellular and humoral immune systems of animals and is an important marker for progenitor B-cell leukemia (Schwarting et al., 1992; Ishida et al., 2003). CD72 is expressed in B cells, where it appears to mediate the interaction between B and T cells. Similarly, CD180 belongs to a family of pathogen receptors known as Toll-like receptors (TLRs). CD180/MD-1 works in concert with TLR4 to control the recognition of $\mathrm{B}$ cells and signaling of lipopolysaccharide, which is a constituent of the cellular membrane of gram-negative bacteria (Miura et al., 1996). Our results indicated that the $C D 1$ gene family, including $C D 1 B$ and $C D 1 D$, was upregulated in line M5.1 whereas $C D 1 D$ was downregulated in line M15.2 (Fig. 4 and Table S3). The lipid antigens in the $C D 1$ family and $C D 2$ activate natural killer $\mathrm{T}(\mathrm{NKT})$ cells via interaction with the T-cell receptor present on the membrane of NKT cells. When activated, NKT cells rapidly produce Th1 and Th2 cytokines via the synthesis of $I F N-\gamma$ and $I L-4$ (Zhou et al., 2006; Bendelac et al., 2007). Furthermore, CD226 is expressed on the surface of NKT cells, monocytes, and a subset of T cells. CD226 is a member of the immunoglobulin superfamily and contains 


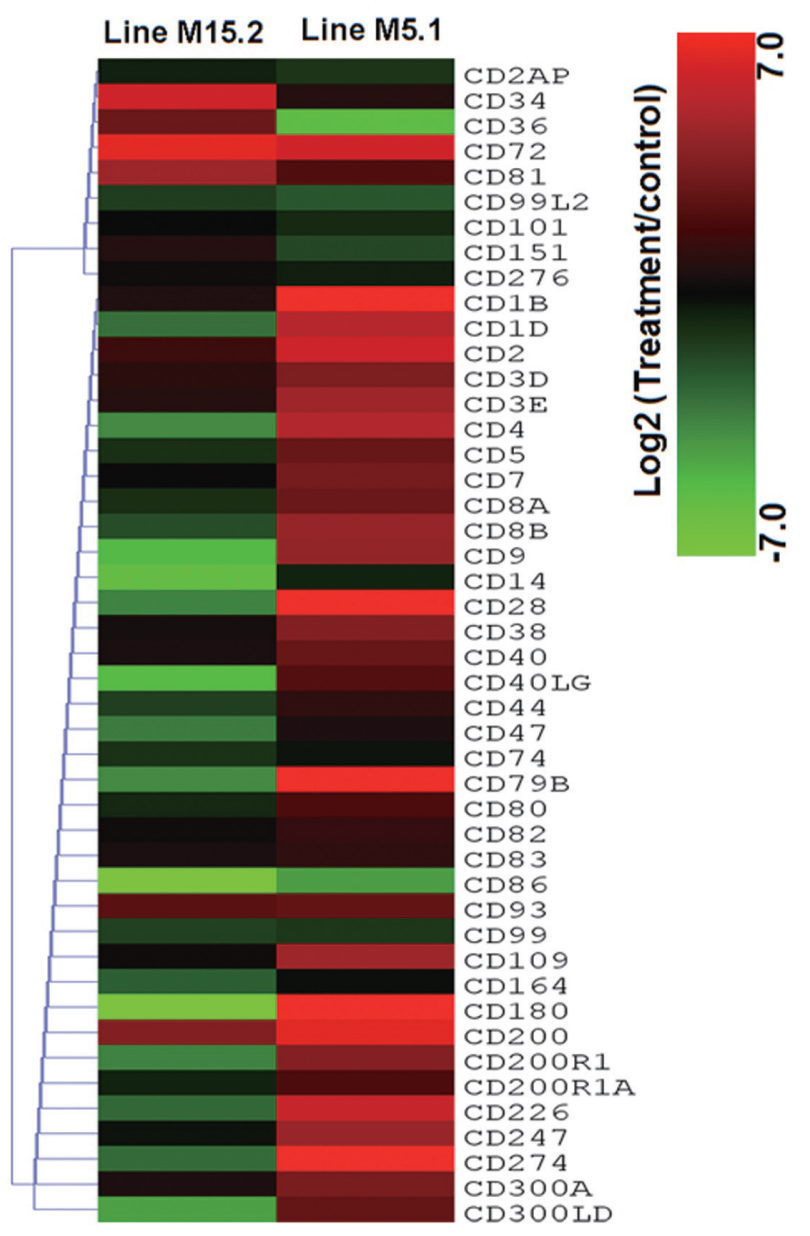

Fig. 4. Expression of $46 \mathrm{CD}$ marker genes in NE induced chicken lines. The heatmap was generated from a hierarchical analysis of the $46 \mathrm{CD}$ marker genes that showed significant changes in the NE induced chicken lines. The genes included here showed significant differences in gene expression $(p<0.01, \log 2$ fold change compared treatment to control) in at least one experiment. The genes shown in red were upregulated expression and those in green were downregulated in the two NE-induced chicken lines.

two Ig-like domains of the V-set (Tahara-Hanaoka et al., 2004). Similar to other cytokines and CD molecules, CD226 was upregulated by 2.44-fold in line M5.1 and was downregulated by 1.12-fold in line M15.2 (Fig. 4 and Table S3). These results indicate that CD molecules are more highly expressed in line M5.1 than in M15.2 and may better regulate the response of innate immune genes to NE in line M5.1 compared with M15.2.

\section{Expression of Immune-related Genes in the NE-induced Chicken Lines}

On the basis of annotation data from the GO Consortium, 89 immune-related genes were identified in this study. To evaluate the host response to NE and the genetic differences between the two chicken lines, the list of immune-related genes was used to narrow the previously identified DEGs ( $p$
$<0.01)$. Using the designated cut-off fold-change of 2.0, four genes in line M15.2 and 15 genes in line M5.1 were found to be differentially expressed after NE induction (Table S4); among them, 11 genes were more highly expressed in line M5.1 than in line M15.2 $(p<0.01$, foldchange $\geq 2$; Table S4). Several of these DEGs play important roles in protein transportation, transmembrane transportation as well as in the interaction between B cells, T-cell immunoglobulin, and BCL2. These genes are probably associated with the degradation and processing of antigens for MHC class I and II molecules (Table S2). Most of the DEGs related to $\mathrm{MHC}$-II antigen-processing pathways, including immunoglobulin, cytokine, and hemoglobin, were significantly upregulated or downregulated (Table S4).

\section{GO Analysis of DEGs}

The analysis of enriched GO terms allowed us to identify significant categories that could be overlooked when evaluating individual genes. The enriched GO terms can help interpret the dominant functions controlled by DEGs (Ashburner et al., 2000). Using GO analysis, approximately 1270 genes with known functions at the logarithm growth phase were identified in Fayoumi line M5.1 and were categorized into 69 terms in the biological processes, in 17 terms in cellular components, and in eight terms in molecular functions. Of the 1270 genes with known functions, 211 genes were assigned to the molecular function category (127 genes were upregulated and 84 genes were downregulated; $p<$ 0.01 , fold-change $\geq 2$ ), 141 genes were assigned to the cellular component category (116 genes were upregulated and 25 genes were downregulated; $p<0.01$, fold-change $\geq 2$ ), and 918 genes were assigned to the biological process category (628 genes were upregulated and 290 genes were downregulated; $p<0.01$, fold-change $\geq 2$; Table S5A). Similarly, GO analysis of line M15.2 suggested that 417 genes with known functions at the logarithm growth phase were categorized into 42 terms in biological processes, in 15 terms in cellular components, and in nine terms in molecular functions, and all the genes with 2-fold changes belonged to the aforementioned categories and were differentially expressed $(p<0.01)$. Of the 417 genes with known functions, 53 genes were assigned to the molecular function category (15 genes were upregulated and 38 genes were downregulated; $p<$ 0.01 , fold-change $\geq 2$ ), 149 genes were assigned to the cellular component category ( 75 genes were upregulated and 74 genes were downregulated; $p<0.01$, fold-change $\geq 2$ ), and 215 genes were assigned to the biological process category (120 genes were upregulated and 95 genes were downregulated; $p<0.01$, fold-change $\geq 2$; Table S5B).

Six major categories in GO's biological process in line M5.1 were associated with signal immune response, singlemulticellular organism process, regulation of locomotion, monocarboxylic acid metabolism, immune processes, and multi-organism processes. However, GO terms in line M15.2 were included in two major clusters: anion transport and immune response (Fig. S2). In addition, two major clusters for cellular component in line M5.1 were associated with contractile fibers and extracellular region, and three major 
Table 2. Distribution of pathways related of two chicken lines with NE induced. KEGG pathway analyses were performed using DAVID Bioinformatics Resources version 6.7, NIAID/NIH (http://david.abcc.ncifcrf.gov/tools.jsp)

\begin{tabular}{|c|c|c|c|c|c|c|c|}
\hline \multirow{2}{*}{ ID } & \multirow{2}{*}{ Pathway } & \multicolumn{3}{|c|}{ Line M5.1 } & \multicolumn{3}{|c|}{ Line M15.2 } \\
\hline & & DEGs & $\%$ & $p$-value & DEGs & $\%$ & $p$-value \\
\hline gga04080 & Neuroactive ligand-receptor interaction & 60 & 3.9 & $5.2 \mathrm{E}-13$ & 51 & 5.4 & $2 \mathrm{E}-17$ \\
\hline gga04060 & Cytokine-cytokine receptor interaction & 40 & 2.6 & 0.00045 & 19 & 2 & 0.013 \\
\hline $\operatorname{gga} 04020$ & Calcium signaling pathway & 33 & 2.2 & 0.000034 & 29 & 3.1 & 5.7E-08 \\
\hline gga04010 & MAPK signaling pathway & 31 & 2 & 0.018 & 11 & 1.2 & 0.039 \\
\hline gga04510 & Focal adhesion & 27 & 1.8 & 0.023 & 14 & 1.5 & 0.024 \\
\hline gga04810 & Regulation of actin cytoskeleton & 27 & 1.8 & 0.049 & 14 & 1.5 & 0.032 \\
\hline gga04514 & Cell adhesion molecules (CAMs) & 22 & 1.4 & 0.0042 & 13 & 1.4 & 0.038 \\
\hline gga04310 & Wnt signaling pathway & 21 & 1.4 & 0.035 & 14 & 1.5 & 0.046 \\
\hline gga04630 & Jak-STAT signaling pathway & 21 & 1.4 & 0.044 & 11 & 1.2 & 0.028 \\
\hline gga04270 & Vascular smooth muscle contraction & 18 & 1.2 & 0.015 & 14 & 1.5 & 0.0047 \\
\hline $\operatorname{gga} 04110$ & Cell cycle & 14 & 0.9 & 0.028 & 3 & 0.3 & 0.099 \\
\hline gga04910 & Insulin signaling pathway & 14 & 0.9 & 0.039 & & & \\
\hline gga04512 & ECM-receptor interaction & 13 & 0.9 & 0.0156 & 10 & 1.1 & 0.028 \\
\hline gga03320 & PPAR signaling pathway & 12 & 0.8 & 0.033 & 4 & 0.4 & 0.01 \\
\hline gga04916 & Melanogenesis & 12 & 0.8 & 0.023 & 14 & 1.5 & 0.0015 \\
\hline gga00590 & Arachidonic acid metabolism & 11 & 0.7 & 0.02 & 2 & 0.2 & 0.095 \\
\hline $\operatorname{gga} 04370$ & VEGF signaling pathway & 11 & 0.7 & 0.011 & 2 & 0.2 & 0.0015 \\
\hline gga04540 & Gap junction & 11 & 0.7 & 0.024 & 14 & 1.5 & 0.00056 \\
\hline $\operatorname{gga} 04350$ & TGF-beta signaling pathway & 10 & 0.7 & 0.034 & 4 & 0.4 & 0.023 \\
\hline gga04012 & ErbB signaling pathway & 9 & 0.6 & 0.048 & 3 & 0.3 & 0.034 \\
\hline gga04914 & Progesterone-mediated oocyte maturation & 9 & 0.6 & 0.047 & & & \\
\hline gga04920 & Adipocytokine signaling pathway & 9 & 0.6 & 0.022 & & & \\
\hline gga00564 & Glycerophospholipid metabolism & 8 & 0.5 & 0.037 & 5 & 0.5 & 0.047 \\
\hline gga04070 & Phosphatidylinositol signaling system & 8 & 0.5 & 0.046 & & & \\
\hline $\operatorname{gga} 00260$ & Glycine, serine and threonine metabolism & 6 & 0.4 & 0.012 & 6 & 0.6 & 0.02 \\
\hline gga00512 & O-Glycan biosynthesis & 6 & 0.4 & 0.011 & 3 & 0.3 & 0.046 \\
\hline gga00601 & Glycosphingolipid biosynthesis-Lacto & 6 & 0.4 & 0.019 & 2 & 0.2 & 0.033 \\
\hline gga03030 & DNA replication & 6 & 0.4 & 0.02 & & & \\
\hline $\operatorname{gga} 04672$ & Intestinal immune network for IgA production & 6 & 0.4 & 0.042 & 5 & 0.5 & 0.024 \\
\hline gga00565 & Ether lipid metabolism & 5 & 0.3 & 0.036 & & & \\
\hline $\operatorname{gga} 00592$ & alpha-Linolenic acid metabolism & 4 & 0.3 & 0.02 & & & \\
\hline gga00603 & Glycosphingolipid biosynthesis & 4 & 0.3 & 0.011 & & & \\
\hline gga04320 & Dorso-ventral axis formation & 4 & 0.3 & 0.037 & & & \\
\hline gga00604 & Glycosphingolipid biosynthesis & 3 & 0.2 & 0.038 & & & \\
\hline gga00910 & Nitrogen metabolism & & & & 4 & 0.4 & 0.011 \\
\hline gga04260 & Cardiac muscle contraction & & & & 7 & 0.7 & 0.021 \\
\hline gga04340 & Hedgehog signaling pathway & & & & 8 & 0.8 & 0.024 \\
\hline
\end{tabular}

clusters for cellular component in line M15.2 were associated with the external plasma membrane, high-density lipoprotein particles, and the extracellular region (Fig. S2). The GO term molecular function had four major clusters in line M5.1: iron-ion binding, gram-positive bacteria binding, chemokine activity, and monooxygenase activity, and four major clusters in line M15.2: organic acid transmembrane transporter activity, transporter activity, lysozyme activity, and integrin binding (Fig. S2). Compared with the results of our previous study (Truong et al., 2015a), GO analysis of Fayoumi chicken and of the inbred chicken lines 6.3 and 7.2 indicated the presence of similar DEGs, and the number of genes that GO enriched categories biological process and cellular component differed between the upregulated and downregulated genes. Therefore, the GO molecular functional enrichment analysis suggests different roles and functions for the genes identified in the two Fayoumi chicken lines.

\section{Pathway Analysis of DEGs}

The pathway analysis was performed to better understand the biological function of the DEGs in the regulatory system. The information on the molecular networks and enriched pathways of the DEGs allowed us to explore the gene network and the underlying molecular mechanisms between the two chicken lines. Using KEGG pathway mapping, 1851 genes were identified (NCBI Gene ID), in which 1270 and 581 DEGs were identified in lines M5.1 and M15.2 respectively; 1175 genes in line M5.1 and 508 genes in line M15.2 were mapped into several KEGG pathways. The most differentially expressed signaling pathways from each group are listed in Table 2. 

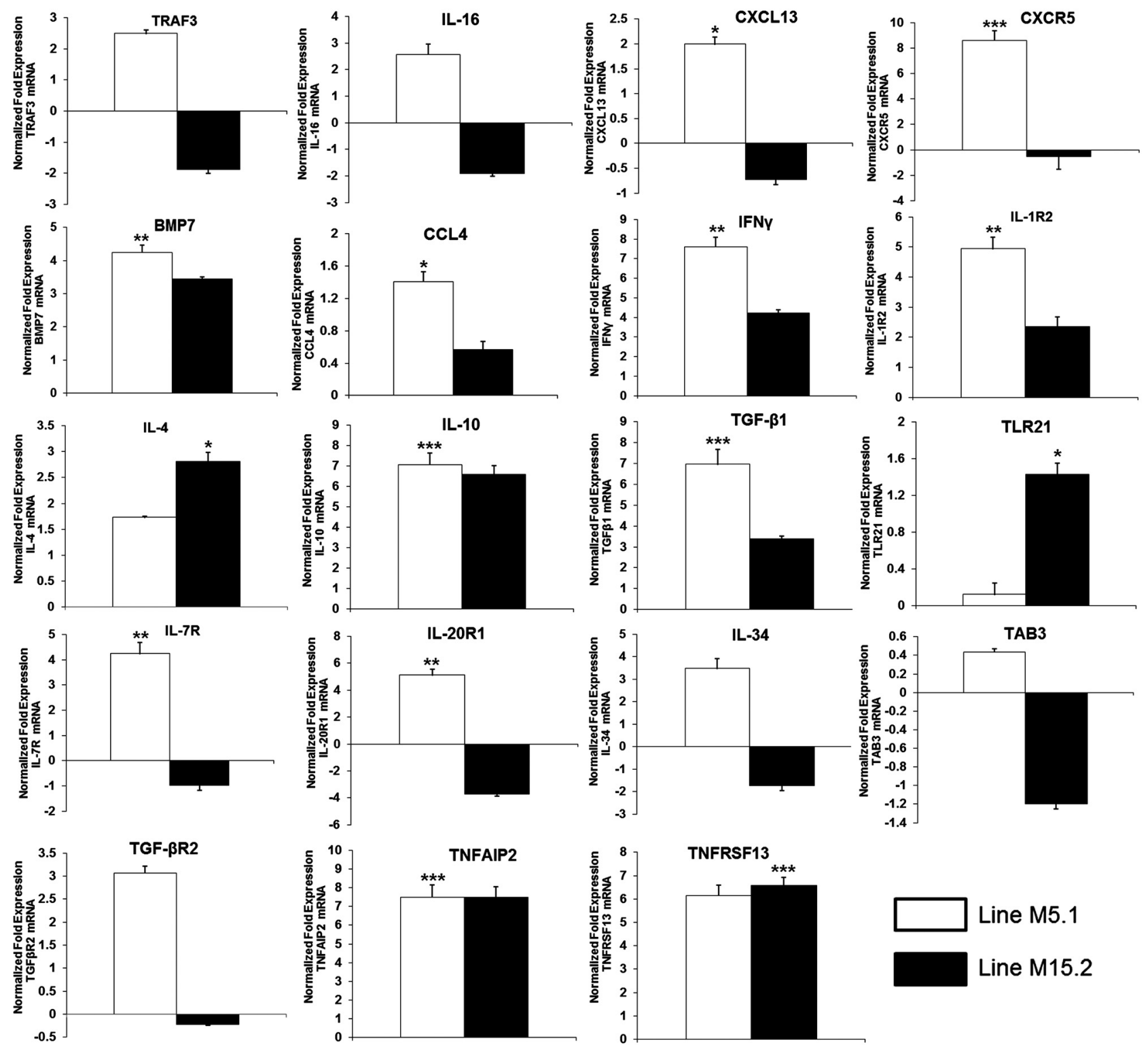

Fig. 5. Quantitative expression analysis of up- and down regulated genes in intestinal mucosa derived from NE induced line M5.1 and M15.2 of Fayoumi chicken. Data were expressed as mean values \pm standard deviation of samples $(n=3)$ and expressed as fold change in mRNA expression: ${ }^{*} p<0.05,{ }^{*} p<0.01$, and ${ }^{* * *} p<0.001$.

These data indicate that the elevated expression of genes in the intestinal mucosa of the infected chickens was strongly associated with immunity and defense (cytokine-cytokine receptor interaction), signal transduction (MAPK, JAKSTAT, ErbB, and phosphatidylinositol signaling pathways), extracellular matrix (ECM) (e.g., focal adhesion, cell adhesion, and ECM-receptor interaction), and cell cycle-associated pathways (apoptosis and cell differentiation). Several upregulated genes are involved in immunological responses, such as those associated with the MAPK and
JAK-STAT signaling pathways, rather than the downregulated genes in each pathway. These results suggest that new populations of immune cells are recruited in the intestine and replace the original population of epithelial cells. Moreover, these observations are consistent with earlier observations that several innate immunity genes that mediate cellular immunity play a key role in the response to NE (Lee et al., 2011).

Many regulated pathways are interconnected or functionally overlap, including cytokine/cytokine receptor interac- 


\section{Chicken line M5.1}

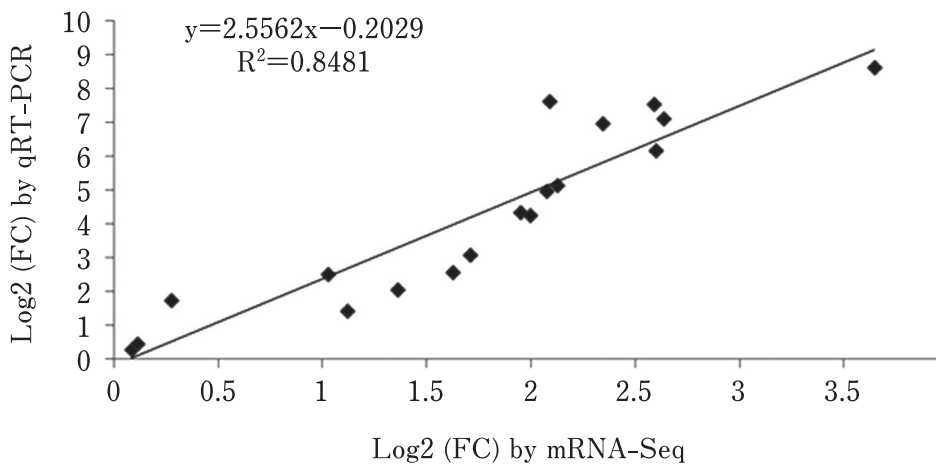

Chicken line M15.2

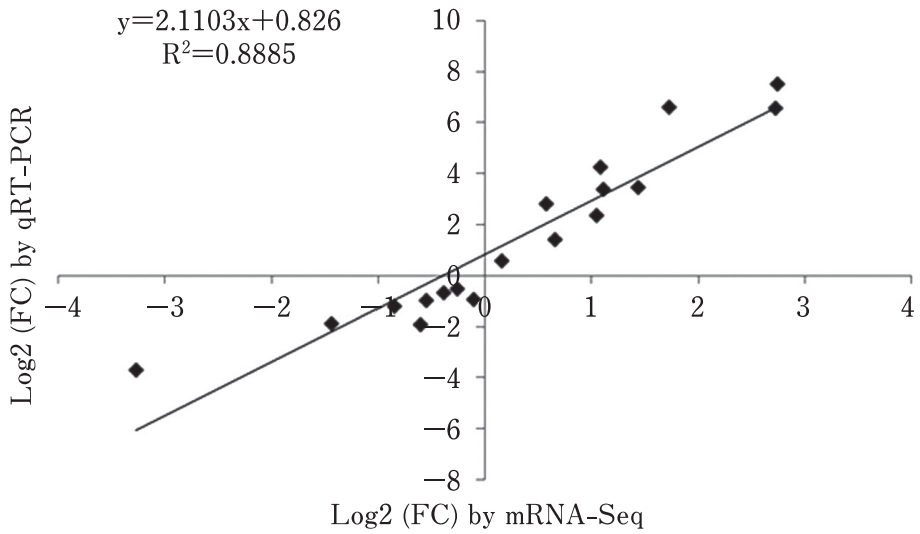

Fig. 6. Significant correlations between expressions of qRT-PCR and RNA-Seq in intestinal mucosa of two genetic chicken lines NE induced.

tions and the JAK-STAT signaling pathway, and immune cells and other cell types are recruited in adhesion molecule interactions, such as those found in MHC class I/II, and TGF$\beta 2$ in dendritic cells; the latter interact with $C D 8, C D 4$, or CD226 in T cells (Huang et al., 2006). Of note, we also observed an increase in the expression of genes that encode $\operatorname{Ig} \mathrm{A}$ in the intestinal immune network, including genes encoding class II MHC in dendritic cells, genes encoding CD28, CD40L, and ICOS in $\mathrm{CD}^{+} \mathrm{T}$ cells, and genes encoding BAFF in epithelial cells (Table 2). Significant levels of IgA were also found in the gut mucosa in response to infection with E. maxima (Lillehoj and Lillehoj, 2000; Min et al., 2013). IgA is a factor involved in intestinal mucosal immunity and plays a key role in protecting the host against infections by pathogens such as Toxoplasma gondii (Bourguin et al., 1991) and Eimeria tenella (Zhou et al., 2015). We hypothesized that IgA production is essential for chickens to develop protective immunity and plays an important role in NE. In both chicken lines, the gene cluster that included neuroactive ligand-receptor interaction, JAKSTAT signaling pathway, MAPK signaling pathway, regulation of actin cytoskeleton, p53 signaling pathway, cytokine- cytokine receptor interaction, and calcium signaling pathway represented the largest cluster, particularly in line M5.1, which is consistent with the response of these chicken lines to NE.

Two genes of the TGF $\beta$ signaling pathway (TGF $\beta 1$ and $T G F \beta 3$ ), which also participate in cytokine-cytokine receptor interaction and in other pathways, including MAPK, mTOR, and JAK-STAT signaling pathways, were upregulated in line M5.1 (Table S2). Most immune cells express TGF $\beta 1$, which plays an important role in the control of the immune system and shows distinct activity profiles in different cell types and developmental stages (Letterio and Roberts, 1998). TGFß3 is involved in the differentiation, embryogenesis, and development of cells (Herpin et al., 2005). The stimulation of skeletal muscle and porcine interstitial cells with TGF 1 increased the mRNA expression of the p38 MAPK family and Mek1/2/Erk1/2 signaling pathways (Das et al., 2013). In this study, the p38 MAPK family (p38 $32, p 38 \alpha, p 38 \gamma$, and $p 38 \delta), J N K 1 / 2 / 3$, and $E R K 1 / 2$ were differentially expressed in the two chicken lines (data not shown). The expression of $T G F \beta 1$ and $T G F \beta 3$ was upregulated by 1.12 - and 3.39 -fold in line M15.2, respectively, and by 2.34- and 1.50-fold in line 
M5.1, respectively, compared with the controls $(p<0.01$; Table S2). Therefore, further studies are required to determine how TGFB1 and TGFB3 are regulated and controlled in the two chicken lines at the cellular level after NE induction. Validation of Differential Gene Expression by $q R T-P C R$

To determine the expression profile obtained using RNASeq analysis, 19 randomly selected DEGs with different levels of expression were used for validation by relative qRT-PCR. The expression levels of these 19 genes are shown in Fig. 5. We also conducted a simple linear regression analysis of the relative expression and calculated the RPKM; the coefficient of determination (or $\mathrm{R}^{2}$ ) was equal to 0.84 for Fayoumi line M5.1 and 0.88 for line M15.2 (Fig. 6). The expression profiles of the selected genes obtained at random using qRT-PCR were consistent with the patterns of expression obtained using RNA-Seq. The results were considered as the technical validation of the DEG analysis.

\section{Conclusion}

In the present study, genome-wide gene expression profiles of the host response to NE induction in the intestinal mucosa of two Fayoumi chicken lines were evaluated using RNA-Seq. We drew four noteworthy conclusions from our results. First, using RNA-Seq, we generated the first draft of approximately 52 and 40 million reads for M5.1 and M15.2 lines, respectively, one of which is a natural host for NE. Second, we used RNA-Seq transcriptome analysis to characterize the DEG profiles and identify genes responsive to NE. Overall, genes of line M5.1 were expressed to a greater extent than those of line M15.2 after NE induction. Third, we detected 89 immune-related and $46 \mathrm{CD}$ molecular genes differentially expressed in the two lines. In addition, approximately 183 innate immune genes were differentially expressed in the two lines. Fourth, we found $13 \beta$-defensin genes with 3 LSDs of AvBD3, and these genes might be involved in the host immune response to NE in these chicken lines. Our results suggest that line M5.1 is more resistant to NE than line 15.2; furthermore, they helped elucidate the mechanisms underlying disease resistance to $\mathrm{NE}$ and will foster the development of effective control strategies against these enteric pathogens.

\section{Conflict of Interest}

The authors have declared that no competing interests exist.

\section{Acknowledgments}

This study was partially supported by the Next-Generation BioGreen 21 Program (No. PJ00808401), Rural Development Administration, and Chung-Ang University Excellent Student Scholarship in 2015, Republic of Korea. We are thank to Dr. Susan J. Lamont (Iowa State University) for Fayoumi chicken.

\section{Sequence Data Availability}

All raw Illumina sequence data can be obtained freely by contacting the Department of Animal Science and Tech- nology, Chung-Ang University. The AMG_Gallus database has been uploaded in the National Agricultural Biotechnology Information Center (NABIC, http://nabic.rda.go.kr/) [ID: NN-1820-000001, NN-1821-000001, NN-1823-000001, and NN-1824-000001].

\section{Supplemental Information}

Fig. S1. Evaluation of sequencing quality. Gene coverage statistics.

Fig. S2. The "TreeMap" view of difference expressed in terms of biological process, cellular component, and molecular function for lines M5.1 and M15.2. Each rectangle is a single cluster representative. The representatives are joined into "superclusters" of loosely related terms and visualized with different colors. Size of the rectangles may be adjusted to reflect $p$-value.

Table S1. Oligonucleotide primers used for quantitative RTPCR.

Table S2. Description of genes responsive to NE induced in the intestinal mucosa of 2 chicken lines. These data show significant changes in gene expression for 183 innate immune genes in the chicken lines. The genes included here show significant differences in gene expression $(p<0.01$, $\log 2$-fold change when treatment was compared with the control).

Table S3. Description of CD molecular genes responsive to $\mathrm{NE}$ induced in the intestinal mucosa of 2 chicken lines. These data show significant changes in gene expression for $46 \mathrm{CD}$ molecular genes in the chicken lines. The genes included here show significant differences in gene expression $(p<$ $0.01, \log 2$-fold change when treatment was compared with the control).

Table S4. Differentially expressed genes related to the antigen processing and presentation pathways in the intestinal mucosa of 2 chicken lines $(p<0.01, \log 2$-fold change when treatment was compared with the control).

Table S5A. Gene ontology (GO) for DEGs in NE-induced line M5.1 (CC: Cellular Component; BP: Biological Process, and MF: Molecular Function; $p<0.01$; log2-fold change when treatment was compared with the control $\geq 2$ or $\leq-2$ ). The $p$ values were calculated using the right-tailed Fisher's exact test. GO functional enrichment analysis was performed using Blast2GO (version 2.3.5) (http://www.blast2go.org/).

Table S5B. Gene ontology (GO) for DEGs in NE-induced line M15.2 (CC: Cellular Component; BP: Biological Process, and MF: Molecular Function; $p<0.01$; log2-fold change when treatment was compared with the control $\geq 2$ or $\leq-2$ ). The $p$ values were calculated using the right-tailed Fisher's exact test. GO functional enrichment analysis was performed using Blast2GO (version 2.3.5) (http://www.blast2go.org/).

\section{References}

Allam JP, Duan Y, Winter J, Stojanovski G, Fronhoffs F, Wenghoefer M, Bieber T, Peng WM and Novak N. Tolerogenic T cells, Th1/Th17 cytokines and TLR2/TLR4 expressing dendritic cells predominate the microenvironment within distinct oral mucosal sites. European Journal of Allergy and Clinical 
Immunology, 66: 532-539. 2011.

Ansari-Lari MA, Muzny DM, Lu J, Lu F, Lilley CE, Spanos S, Malley T and Gibbs RA. A gene-rich cluster between the CD4 and triosephosphate isomerase genes at human chromosome 12p13. Genome Research, 6: 314-326. 1996.

Ashburner M, Ball CA, Blake JA, Botstei D, Butler H, Cherry JM, Davis AP, Dolinski K, Dwight, SS, Eppig JT, Harris MA, Hill DP, Issel-Tarver L, Kasarskis A, Lewis S, Matese JC, Richardson JE, Ringwald M, Rubin GM and Sherlock G. Gene ontology: tool for the unification of biology. The Gene Ontology Consortium, Nature Genetics, 25: 25-29. 2000.

Bendelac A, Savage PB and Teyton L. The biology of NKT cells. Annual Review of Immunology, 25: 297-336. 2007.

Bourguin I, Chardes T, Mevelec MN, Woodman JP and Bout D. Amplification of the secretory IgA response to Toxoplasma gondii using cholera toxin. FEMS Microbiology Letters, 65: 265-271. 1991.

Chitwood JL, Rincon G, Kaiser GG, Medran JF and Ross PJ. RNAseq analysis of single bovine blastocysts. BMC Genomics, 14: 350. 2013.

Das D, Holmes A, Murphy GA, Mishra K, Rosenkranz AC, Horowitz JD and Kennedy JA. TGF-beta1-Induced MAPK activation promotes collagen synthesis, nodule formation, redox stress and cellular senescence in porcine aortic valve interstitial cells. Journal of Heart Valve Disease, 22: 621-630. 2013.

Herpin A, Lelong C, Becker T, Rosa FM, Favrel P and Cunningham C. Structural and functional evidences for a type 1 TGF-beta sensu stricto receptor in the lophotrochozoan Crassostrea gigas suggest conserved molecular mechanisms controlling mesodermal patterning across bilateria. Mechanisms of Development, 122: 695-705. 2005.

Hong YH, Song W, Lee SH and Lillehoj HS. Differential gene expression profiles of beta-defensins in the crop, intestine, and spleen using a necrotic enteritis model in 2 commercial broiler chicken lines. Poultry Science, 91: 1081-1088. 2012.

Huang R, Wallqvist A and Covell DG. Comprehensive analysis of pathway or functionally related gene expression in the National Cancer Institute's anticancer screen. Genomics, 87: 315-328. 2006.

Huang Y, Li Y, Burt DW, Chen H, Zhang Y, Qian W, Kim H, Gan S, Zhao Y, Li J, Yi K, Feng H, Zhu P, Li B, Liu Q, Fairley S, Magor KE, Du Z, Hu X, Goodman L, Tafer H, Vignal A, Lee T, Kim KW, Sheng Z, An Y, Searle S, Herrero J, Groenen MA, Crooijmans RP, Faraut T, Cai Q, Webster RG, Aldridge JR, Warren WC, Bartschat S, Kehr S, Marz M, Stadler PF, Smith J, Kraus RH, Zhao Y, Ren L, Fei J, Morisson M, Kaiser P, Griffin DK, Rao M, Pitel F, Wang J and Li N. The duck genome and transcriptome provide insight into an avian influenza virus reservoir species. Nature Genetics, 45: 776-783. 2013.

Ishida I, Kumanogoh A, Suzuki K, Akahani S, Noda K and Kikutani H. Involvement of CD100, a lymphocyte semaphorin, in the activation of the human immune system via CD72: implications for the regulation of immune and inflammatory responses. International Immunology, 15: 1027-1034. 2003.

Kaiser P, Poh TY, Rothwell L, Avery S, Balu S, Pathania US, Hughes S, Goodchild M, Morrell S, Watson M, Bumstead N, Kaufman J and Young JR. A genomic analysis of chicken cytokines and chemokines. Journal of Interferon \& Cytokine Research, 25: 467-484. 2005.

Khawli LA, Hu P and Epstein AL. Cytokine, chemokine, and costimulatory fusion proteins for the immunotherapy of solid tumors. Handbook of Experimental Pharmacology, 181: 291-
328. 2008.

Kim DK, Lillehoj HS, Hong YH, Park DW, Lamont SJ, Han JY and Lillehoj EP. Immune-related gene expression in two B-complex disparate genetically inbred Fayoumi chicken lines following Eimeria maxima infection. Poultry Science, 87: 433-443. 2008.

Kim DK, Kim CH, Lamont SJ, Keeler CL and Lillehoj HS. Gene expression profiles of two B-complex disparate, genetically inbred Fayoumi chicken lines that differ in susceptibility to Eimeria maxima. Poultry Science, 88: 1565-1579. 2009.

Kim DK, Lillehoj HS, Jang SI, Lee SH, Hong YH and Lamont SJ. Genetically Disparate Fayoumi chicken lines show different response to avian necrotic enteritis. Journal of Poultry Science, 52: 245-252. 2015.

Lee JH, Gao C, Peng G, Greer C, Ren S, Wang Y and Xiao X. Analysis of transcriptome complexity through RNA sequencing in normal and failing murine hearts. Circulation Research, 109: 1332-1341. 2012.

Lee KW, Lillehoj HS, Jeong W, Jeoung HY and An DJ. Avian necrotic enteritis: experimental models, host immunity, pathogenesis, risk factors, and vaccine development. Poultry Science, 90: 1381-1390. 2011.

Lehrer RI and Ganz, T. Defensins of vertebrate animals. Current Opinion in Immunology, 14: 96-102. 2002.

Letterio JJ and Roberts AB. Regulation of immune responses by TGF- $\beta$. Annual Review of Immunology, 16: 137-161. 1998.

Lillehoj HS and Lillehoj EP. Avian coccidiosis: A review of acquired intestinal immunity and vaccination strategies. Avian Diseases, 44: 408-425. 2000.

Lister R, O’Malley RC, Tonti-Filippini J, Gregory BD, Berry CC, Millar AH and Ecker JR. Highly integrated single-base resolution maps of the epigenome in Arabidopsis. Cell, 133: 523536. 2008.

Livak KJ and Schmittgen TD. Analysis of relative gene expression data using real-time quantitative PCR and the $2^{-\Delta \Delta C T}$ Method. Methods, 25: 402-408. 2001.

Lynn DJ, Higgs R, Lloyd AT, O'Farrelly C, Herve-Grepinet V, Nys Y, Brinkman FS, Yu PL, Soulier A, Kaiser P, Zhang G and Lehrer RI. Avian $\beta$-defensin nomenclature: a community proposed update. Immunology Letters, 110: 86-89. 2007.

McReynolds J, Waneck C, Byrd J, Genovese K, Duke S and Nisbet D. Efficacy of multistrain direct-fed microbial and phytogenetic products in reducing necrotic enteritis in commercial broilers. Poultry Science, 88: 2075-2080. 2009.

Min W, Kim WH, Lillehoj EP and Lillehoj HS. Recent progress in host immunity to avian coccidiosis: IL-17 family cytokines as sentinels of the intestinal mucosa. Developmental and Comparative Immunology, 41: 418-428. 2013.

Miura Y, Miyake K, Yamashita Y, Shimazu R, Copeland NG, Gilbert DJ, Jenkins NA, Inazawa $J$, Abe $T$ and Kimoto $M$. Molecular cloning of a human RP105 homologue and chromosomal localization of the mouse and human RP105 genes (Ly64 and LY64). Genomics, 38: 299-304. 1996.

Mortazavi A, Williams BA, McCue K, Schaeffer L and Wold B. Mapping and quantifying mammalian transcriptomes by RNASeq. Nature Methods, 5: 621-628. 2008.

Mot D, Timbermont L, Delezie E, Haesebrouck F, Ducatelle R and Van Immerseel F. Day-of-hatch vaccination is not protective against necrotic enteritis in broiler chickens. Avian Pathology, 42: 179-184. 2013.

Parish WE. Necrotic enteritis in the fowl (Gallus gallus domesticus) I Histopathology of the disease and isolation of a strain of 
Clostridium welchii. Journal of Comparative Pathology, 71: 377-393. 1961.

Pinard-Van Der Laan MH, Monvoisin JL, Pery P, Hamet N and Thomas M. Comparison of outbred lines of chickens for resistance to experimental infection with coccidiosis (Eimeria tenella). Poultry Science, 77: 185-191. 1998.

Robinson MD, McCarthy DJ and Smyth GK. edgeR: A Bioconductor package for differential expression analysis of digital gene expression data. Bioinformatics, 26: 139-140. 2010.

Schwarting R, Castello R, Moldenhauer G, Pezzutto A, von Hoegen I, Ludwig WD, Parnes JR and Dorken B. Human Lyb-2 homolog CD72 is a marker for progenitor B-cell leukemias. American Journal of Hematology, 41: 151-158. 1992.

Stefanova I and Horejsi V. Association of the CD59 and CD55 cell surface glycoproteins with other membrane molecules. Journal of Immunology, 147: 1587-1592. 1991.

Sugiarto $\mathrm{H}$ and $\mathrm{Yu}$ PL. Avian antimicrobial peptides: the defense role of $\beta$-defensins. Biochemical and Biophysical Research Communications, 323: 721-727. 2004.

Suzawa K, Kobayashi M, Sakai Y, Hoshino H, Watanabe M, Harada O, Ohtani H, Fukuda M and Nakayama J. Preferential induction of peripheral lymph node addressin on high endothelial venule-like vessels in the active phase of ulcerative colitis. The American Journal of Gastroenterology, 102: 1499-1509. 2007.

Tahara-Hanaoka S, Shibuya K, Onoda Y, Zhang H, Yamazaki S, Miyamoto A, Honda S, Lanier LL and Shibuya A. Functional characterization of DNAM-1 (CD226) interaction with its ligands PVR (CD155) and nectin-2 (PRR-2/CD112). International immunology, 16: 533-538. 2004.

Trapnell C, Williams BA, Pertea G, Mortazavi A, Kwan G, van Baren MJ, Salzberg SL, Wold BJ and Pachter L. Transcript assembly and quantification by RNA-Seq reveals unannotated transcripts and isoform switching during cell differentiation. Nature Biotechnology, 28: 511-515. 2010.
Trapnell C, Roberts A, Goff L, Pertea G, Kim D, Kelley DR, Pimentel H, Salzberg SL, Rinn JL and Pachter L. Differential gene and transcript expression analysis of RNA-seq experiments with TopHat and Cufflinks. Nature Protocols, 7: 562578. 2012.

Truong $\mathrm{AD}$, Hong $\mathrm{YH}$ and Lillehoj HS. High-throughput sequencing reveals differing immune responses in the intestinal mucosa of two inbred lines afflicted with necrotic enteritis. Veterinary Immunology and Immunopathology, 166: 116-124. 2015a.

Truong AD, Hong YH and Lillehoj HS. RNA-seq Profiles of Immune Related Genes in the Spleen of Necrotic Enteritisafflicted Chicken Lines. Asian-Australasian Journal of Animal Sciences, 28: 1496-1511. 2015b.

van Dijk A, Veldhuizen EJ and Haagsman HP. Avian defensins. Veterinary Immunology and Immunopathology, 124: 1-18. 2008.

Wang W, Qin Z, Feng Z, Wang X and Zhang X. Identifying differentially spliced genes from two groups of RNA-seq samples. Gene, 518: 164-170. 2013.

Zhou BH, Liu LL, Liu J, Yuan FW, Tian EJ and Wang HW. Effect of diclazuril on the bursa of Fabricius morphology and SIgA expression in chickens infected with Eimeria tenella. Korean Journal of Parasitology, 53: 675-682 2015.

Zhou D. The immunological function of iGb3. Current Protein \& Peptide Science, 7: 325-333. 2006.

Zhou H and Lamont SJ. Genetic characterization of biodiversity in highly inbred chicken lines by microsatellite markers. Animal Genetics, 30: 256-264. 1999.

Zola H, Swart B, Banham A, Barry S, Beare A, Bensussan A, Boumsell L, D Buckley C, Buhring HJ, Clark G, Engel P, Fo, D, Jin BQ, Macardle PJ, Malavasi F, Mason D, Stockinger H and Yang X. CD molecules 2006-human cell differentiation molecules. Journal of Immunological Methods, 319: 1-5. 2007. 